\title{
Statistical analysis of the parameters of the optic disk image
}

\author{
Valery Bakutkin ${ }^{1}$, Vadim Gorelov ${ }^{2}$, Yuriy Zayko ${ }^{2 *}$ \\ ${ }^{1}$ Saratov Research Institute of Rural Hygiene, 1A Zarechnaya str., Saratov 410022, Russia \\ ${ }^{2}$ Stolypin Volga Region Institute of Administration, Russian Presidential Academy of National Economy and Public \\ Administration, 23/25 Sobornaya str., Saratov 410031, Russia \\ *e-mail: yuriyz@mail.ru
}

\begin{abstract}
In recent time much attention is payed to the computer analysis of the optic disc (OD) images, aimed at extracting the objective statistical characteristics of the OD image. In the present paper we describe a computer program that allows the determination of the mean colour indices, their variance, etc., for different regions of the OD. The total colour space in the RGB model is divided into subdomains in correspondence with the values of the mask that determines the subdomain boundaries. A simple method of determining the domain of the OD physiological excavation by the value of the variability coefficient is proposed. (C) 2016 Samara State Aerospace University (SSAU).
\end{abstract}

Keywords: eye diseases, optical disc, computer program.

Paper \#2730 received 2015.11.15; revised manuscript received 2015.12.05; accepted for publication 2015.12.30; published online 2016.02.16.

\section{References}

1. V. V .Bakutkin, Y. N. Zayko, A. S. Lepesko, and A. N. Skidanov, "Development of a system for classification and diagnostics of ophthalmologic diseases based on artificial neuron networks," Proc. of Saratov University, Ser. Physics 8(1), 36-41 (2008).

2. V. V. Bakutkin, V. V. Gorelov, and Y. N. Zayko, "Computer analysis of the optic disk image," 19th School for Junior Scientists and Students on Optics, Laser Physics \& Biophotonics, Saratov, Russia, 21-25 September 2015.

3. Y. N. Zayko, "Physics of Color Formation," Journal of Applied Physical Science International 2(4), 137-144 (2015).

4. G. Wyszecki, and W. Stiles, Color Science. Concepts and Methods, Quantitative Data and Formulas, 1st ed., Willey (1967). ISBN: 978-0471969204.

5. V. V. Gorelov, "Development of methods for analyzing medical data (by the example of visual nerve images)," Diploma project / Supervised by Zayko Yu. N., Stolypin Volga Region Institute of Administration, Saratov (2010).

6. B. Y. Kuritsky, Search for Optimal Solutions Using Excel 7.0 in Examples, BHV (1997). ISBN: 5-7791-0037-3.

7. V. A. Machehin, "Modern techniques in the analysis of the disc of the optic nerve," Russian Medical Journal. Clinical ophthalmology. Glaucoma 1, 14-18 (2011).

8. V. V. Bakutkin, Y. A. Dubinina, and I. O. Kolbenev, "Method of diagnostics of the visual nerve atrophy," The patent of the Russian Federation №2282390 from 27.08.2006, Patent application №20005102366, priority of 31.01.2005.

9. Y. A. Dubinina, "Complex system of assessing the optic disc condition in patients with primary open-angle glaucoma," Abstract of the Candidate of Medical Sciences thesis, Samara State Medical University, Samara (2011).

10. Y. A. Dubinina, V. V. Bakutkin, and I. O. Kolbenev, "Color index in the diagnostics of partial atrophy of optic nerve of various aetiology," Theses of Reports, VIII Congress of Ophthalmologists of Russia, Moscow, Russia, 1-4 June 2005, 692-693 (2005).

11. V. V. Bakutkin, I. V. Bakutkin, Y. N. Zayko, and V. F. Spirin, "Computer image analysis of the optic nerve in clinical practice," Proc. of the Orenburg State University 4(153), 27-29 (2013). 


\section{Introduction}

The present work was carried out within the framework of the Agreement about the scientific and technical collaboration between the Stolypin Volga Region Institute of Administration and the Research Institute of Rural Hygiene (Saratov) and was aimed at the development of a physical approach to the colourimetric analysis of the human eye images. The beginning of the development of the physical theory of colour perception was started from 2006 and is related to the use of artificial neuron networks in the diagnostics of eye diseases [1]. Later efforts were mainly aimed at the use of computer analysis of the optic disc (OD) images in order to get objective quantitative characteristics of glaucoma [2]. An alternative branch of studies was related to the development of statistical theory of colour formation and perception [3] basing on the concept of colour temperature (CT) of the emitting object. All existing methods of the CT determination are associated with using the concept of the "standard colourimetric observer" [4], which introduces uncertainty into the colourimetric measurements. The method of CT measurement free of this drawback is described in [3].

Another source of uncertainty in colourimetric measurements implemented using the computer images is due to the properties of standard monitors. These measurements require corrections that should account for the specific features mentioned above. However, if the images are obtained and stored in the computer memory in a certain format, the correction is not necessary, since all computer programs "see" the same file that contains all information about the colour composition of each pixel.

This approach became possible, because in the recent time many programs operating with the computer images appeared, particularly in medicine. All problems related to the correct colour reproduction are solved at the stage of the image preparation before recording the file.

In this paper we present the results of the OD images, obtained using the special instrument, the "OPTON" fundus-camera. It is a combination of a microscope and a photo camera. The scanning method is known as the eye fundus photorecording. The experimental material offered by the Research Institute of Rural Hygiene (Saratov) was obtained in patients without expressed signs of glaucoma. The study was aimed at clarifying the boundary between the norm and the glaucoma pathology. The method of study was the statistical analysis of the OD. The object of study were the OD images obtained in a number of patients belonging to different age groups and having no expressed signs of glaucoma.

\section{Measurement technique}

To achieve the formulated goal, we developed a computer program that determined the mean colour characteristics and mean-square variance for different regions of OD. Figure 1 shows the program interface.

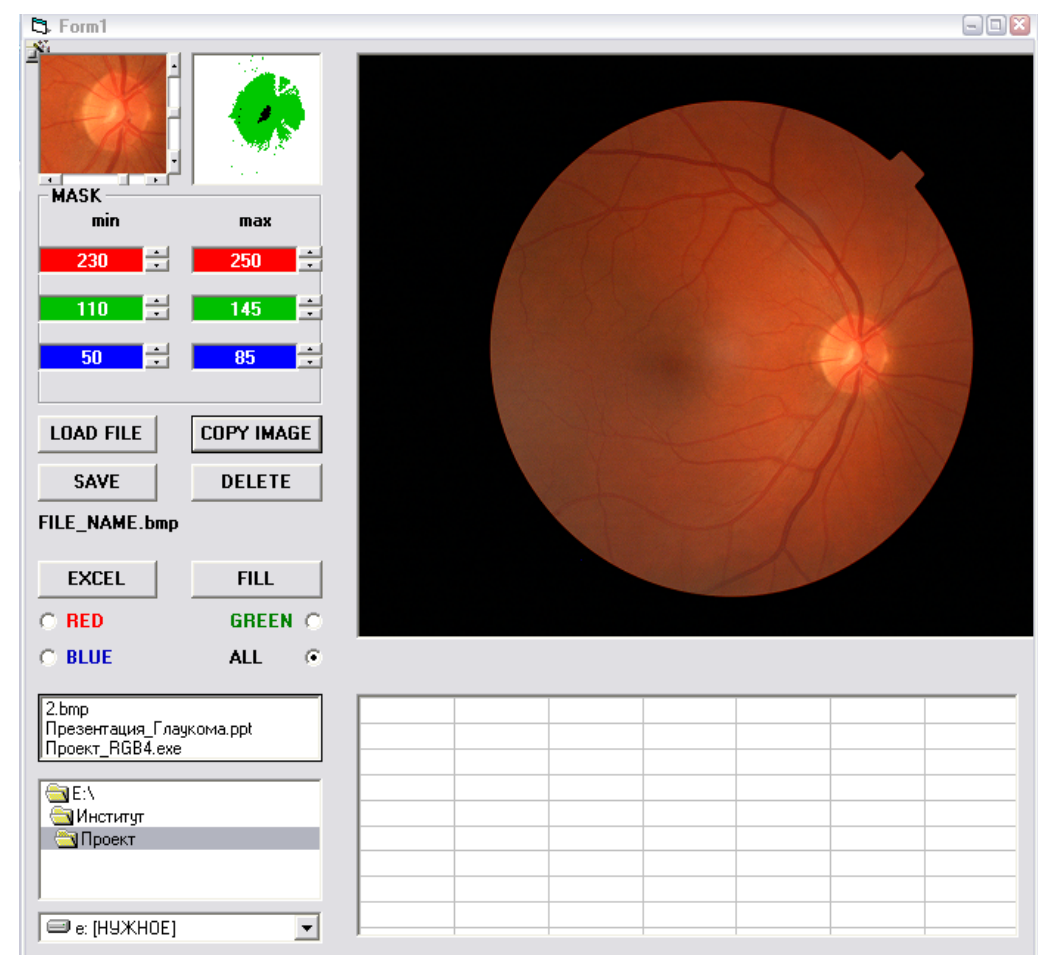

Fig. 1 The program interface. The top right window is the initial image; the small top left windows display the OD area and the result of its processing with the program. Below them the windows for the input of the colour mask values are seen. The rest elements are used for the interface between the program and the environment. 


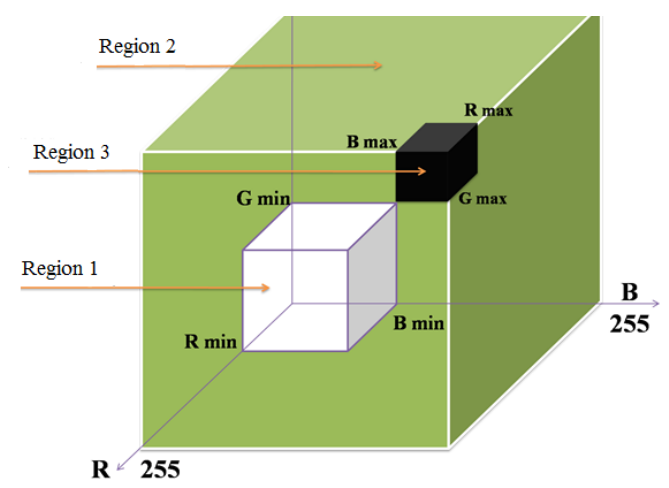

Fig. 2 Division of the RGB colour space into three subdomains.

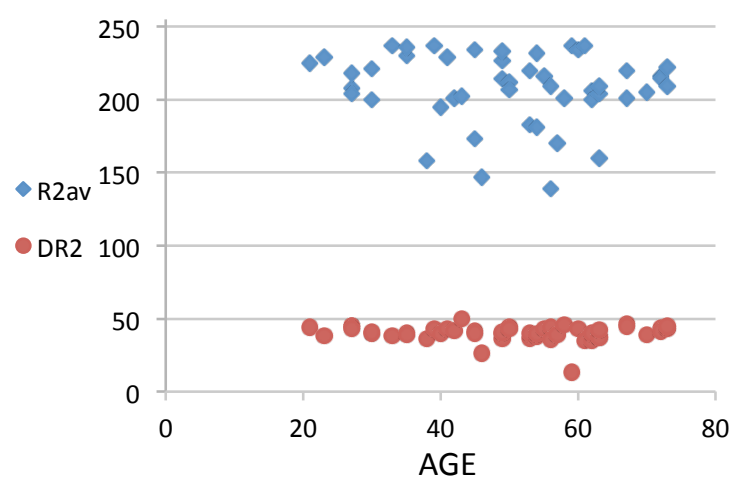

Fig. 3 Dependence of R2av and DR2 (ordinate) upon the age of the patients (abscissa).

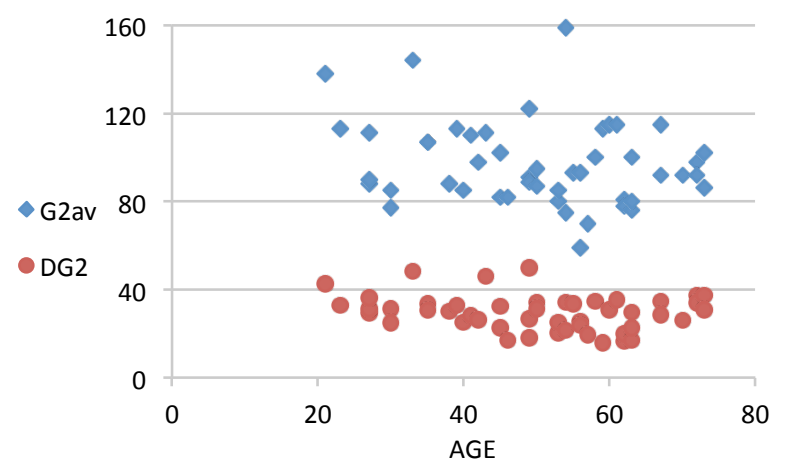

Fig. 4 Dependence of G2av and DG2 (ordinate) upon the age of the patients (abscissa).

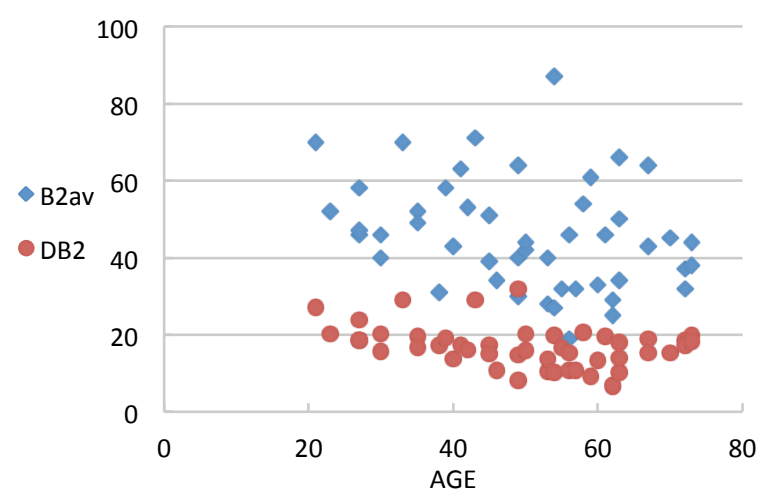

Fig. 5 Dependence of B2av and DB2 (ordinate) upon the age of the patients (abscissa).

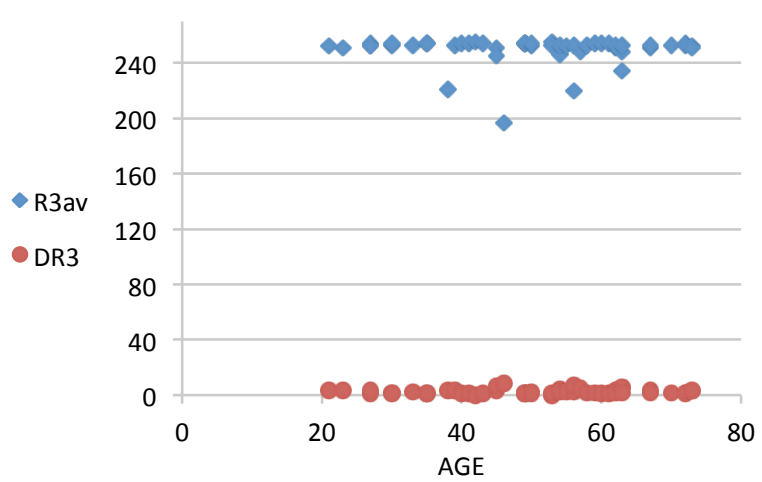

Fig. 6 Dependence of R3av and DR3 (ordinate) upon the age of the patients (abscissa).

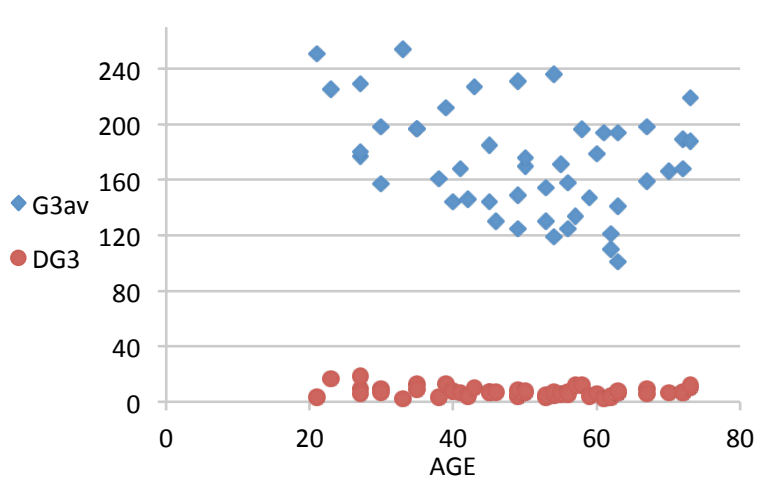

Fig. 7 Dependence of G3av and DG3 (ordinate) upon the age of the patients (abscissa).

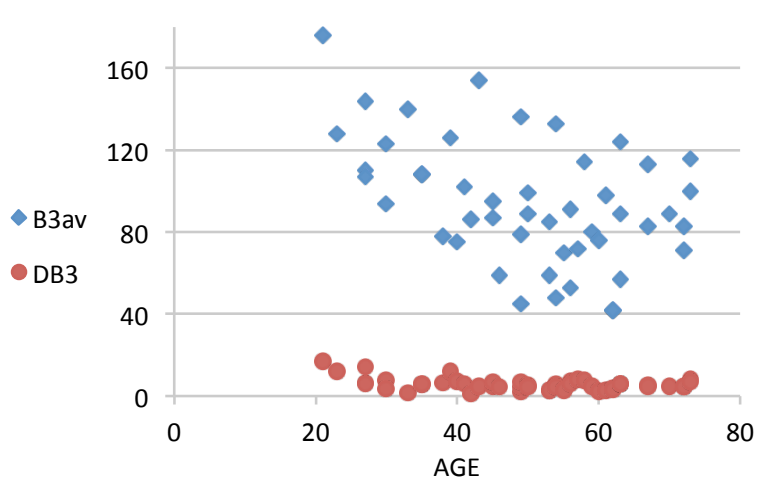

Fig. 8 Dependence of B3av and DB3 (ordinate) upon the age of the patients (abscissa).

According to the developed algorithm the OD domain is divided into three subdomains by applying a special mask, the role of which is to separate the pixels depending on their colour composition. For the RGB version of the program (the program can support the measurements in the HSV model as well) the pixels, for which the values of the red, green, and blue components satisfy the inequalities $R<R_{\mathrm{MIN}}, G<G_{\mathrm{MIN}}$ and $B<B_{\mathrm{MIN}}$, are attributed to the subdomain 1 (beyond the OD in Fig. 1 it is shown in white). The pixels for which $R>R_{\mathrm{MAX}}, G>G_{\mathrm{MAX}}$ and $B>B_{\mathrm{MAX}}$ belong to the subdomain 3 (the domain of physiological excavation of the OD, in Fig. 1 shown in black). All the rest pixels form the subdomain 2 ("healthy" region of the OD, in 
Fig. 1 shown in green colour). The boundary values $R_{\mathrm{MAX}, \mathrm{MIN}}, G_{\mathrm{MAX}, \mathrm{MIN}}$, and $B_{\mathrm{MAX}, \mathrm{MIN}}$ are determined by preliminary pixel-by-pixel scanning of the initial image in the top right window (Fig. 1). Figure 2 shows the result of the division of the RGB colour space into three subdomains depending on the mask values $R_{\mathrm{MIN}}, G_{\mathrm{MIN}}$, $B_{\mathrm{MIN}}$ and $R_{\mathrm{MAX}}, G_{\mathrm{MAX}}, B_{\mathrm{MAX}}$.

The colourimetric statistical analysis of the OD includes the determination of the mean values of Rav, Gav, Bav in the subdomains 2 and 3 of the OD using the program, as well as the calculation of the relevant rootmean-square deviations $D R, D G, D B$. The measurement performed in the groups of patients of different age without expressed signs of glaucoma were aimed at the determination of the boundaries between the norm and pathology for this disease. The results of the analysis are presented in Figs. 3-8 [5].

\section{Discussion}

As mentioned above, the main goal of the studies was to find a simple criterion for the determination of the domain of the physiological excavation of OD. This is just the domain, in which the phenomena accompanying the glaucoma development are observed. For this aim it is convenient to use the so called variability coefficient [6]: $\boldsymbol{V}_{\boldsymbol{X N}}=\boldsymbol{D} \boldsymbol{X} \boldsymbol{N} / \boldsymbol{X} \boldsymbol{N}_{\boldsymbol{a v}}$, where $X=R, G, B$ are the colour components, and $N=2,3$ is the number of the subdomain. As shown by the calculations, the value of the variability coefficient $V X 2$ for the domain conditionally considered as healthy exceeds by more than an order of magnitude the value of the analogous quantity for the domain of physiological excavation $V X 3$. The corresponding results of the calculations for the red component $(X=R)$ are shown in Fig. 9. For other colour components, the green $(X=G)$ and the blue one $(X=B)$, the corresponding results demonstrate similar behavior.

As follows from the papers [7-10], similar methods of optic disk analysis are used in the diagnostics of wide spectrum of eye diseases.

The authors of Ref. [7] describe the morphometric analysis of the OD parameters using the laser retina tomograph Heidelberg Engineering Company (HRT). Having appeared at the market of ophthalmological instruments in the middle of 1990 s, it promoted the OD examination to the level of accuracy, comparable with that of the computer examination of the central visual field. During the last 10-15 years the Russian and foreign ophthalmologists carried out numerous complex studies of the capabilities of this method in the eyes of healthy persons with normal refraction and myopia, in patients with suspicion of glaucoma, and with different stages of low-pressure glaucoma.

In Refs. $[8,9,10]$ the authors propose a method for diagnosing the visual nerve atrophy basing on the eye fundus imaging, extracting the $\mathrm{OD}$ region, and analysing its image by means of the computer system. The system includes the fundus camera "OPTON", the high-resolution colour video camera, the softwarehardware complex for image digitising and the software for the image analysis. The developed set of indicators for assessing the presence and the degree of the OD decolouration provides the opportunity of the objective assessment of the OD structural changes at the expense of using the calculated integral index in absolute units that allows for the distribution of the values of basic spectral components and does not depend upon the imaging conditions.
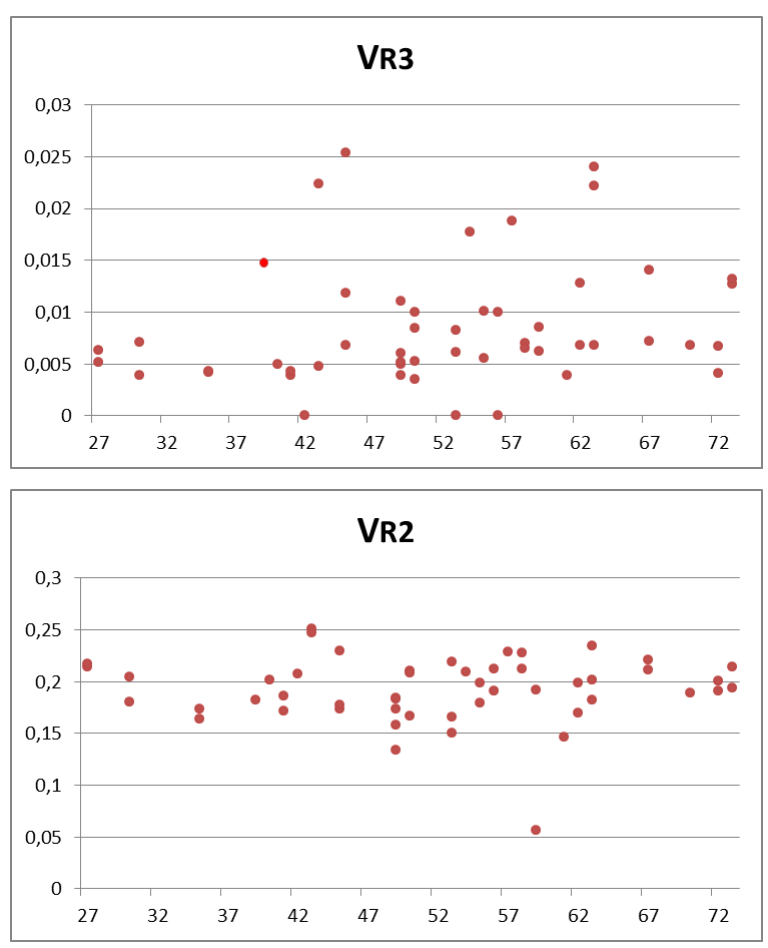

Fig. 9 The values of variability coefficient (ordinate) for the red component in the subdomains 2 (bottom) and 3 (top) for the OD versus the age of the patients (abscissa).

The technique described in the present paper allows the increase of precision and rate of getting objective colour characteristics of the OD and at present has already found application in the clinical practice [11].

\section{Conclusion}

The paper is devoted to the statistical analysis of the optical disc (OD) image parameters, carried out using a specially developed computer program. We present a brief description of the operation of the program, the calculation technique, and the obtained results. It is shown that one can definitely select the region of physiological excavation of OD by the value of the variability coefficient of the $\mathrm{R}, \mathrm{G}$, or $\mathrm{B}$ colour component, which becomes strongly reduced within the above region.

\section{Acknowledgments}

The authors express gratitude to Spirin V. F. and Bakutkin I. V. for collaboration at the stage of clinical tests. 